\title{
Attentional Bias for Non-drug Reward is Magnified in Addiction
}

\author{
Brian A. Anderson, M.S. ${ }^{1}$, Monica L. Faulkner, M.A. ${ }^{2}$, Jessica J. Rilee, B.A. ${ }^{2}$, Steven Yantis, \\ Ph.D. ${ }^{1}$, and Cherie L. Marvel, Ph.D. ${ }^{2,3}$ \\ ${ }^{1}$ Department of Psychological and Brain Sciences, Johns Hopkins University, Baltimore, MD \\ 21218 \\ 2Department of Neurology, Division of Cognitive Neuroscience, Johns Hopkins University, \\ Baltimore, MD 21205 \\ ${ }^{3}$ Department of Psychiatry and Behavioral Sciences, Johns Hopkins University, Baltimore, MD \\ 21205
}

\begin{abstract}
Attentional biases for drug-related stimuli play a prominent role in addiction, predicting treatment outcome. Attentional biases also develop for stimuli that have been paired with non-drug reward in adults without a history of addiction, the magnitude of which is predicted by visual working memory capacity and impulsiveness. We tested the hypothesis that addiction is associated with an increased attentional bias for non-drug (monetary) reward relative to that of healthy controls, and that this bias is related to working memory impairments and increased impulsiveness. Seventeen patients receiving methadone maintenance treatment for opioid dependence and seventeen healthy controls participated. Impulsiveness was measured using the Barratt Impulsiveness Scale (BIS-11), visual working memory capacity was measured as the ability to recognize briefly presented color stimuli, and attentional bias was measured as the magnitude of response time slowing caused by irrelevant but previously reward-associated distractors in a visual search task. The results showed that attention was biased toward the distractors across all participants, replicating previous findings. Importantly, this bias was significantly greater in the patients than in the controls and was negatively correlated with visual working memory capacity. Patients were also significantly more impulsive than controls as a group. Our findings demonstrate that patients in treatment for addiction experience greater difficulty ignoring stimuli associated with non-drug reward. This non-specific reward-related bias could mediate the distracting quality of drug-related stimuli previously observed in addiction.
\end{abstract}

\section{Keywords}

attention; reward; learning; working memory; impulsiveness

It has been well documented that patients in treatment for addiction involuntarily orient attention toward stimuli that are associated with their substance of abuse, whereas individuals with no history of substance abuse do not show such selection biases (Field \& Cox, 2008; Lubman, Peters, Mogg, Bradley, \& Deakin, 2000; Mogg, Bradley, Field, \& De Houwer, 2003; Nickolaou, Field, Critchley, \& Duka, 2013; Nickolaou, Field, \& Duka, 2013;

To whom correspondence should be addressed: Brian A. Anderson, Johns Hopkins University, Psychological \& Brain Sciences, 3400 N. Charles St., Baltimore, MD 21218-2686, bander33@jhu.edu Phone: (717) 725-5366, Fax: (410) 516-4478.

Disclosures

All authors made substantive contributions and have read and approved the final manuscript.

The authors declare no conflicts of interest. 
Stromark, Field, Hugdahl, \& Horowitz, 1997). Similar attentional biases are also evident in heavy but non-dependent substance users (Field, Mogg, Zetteler, \& Bradley, 2004;

Townshend \& Duka, 2001). This bias in attentional orienting is believed to facilitate drug seeking and consumption (Berridge, 2012; Berridge \& Robbinson, 1998). Consistent with a close relationship between addiction and attention, patients who show the largest attentional biases for drug-related stimuli are the most likely to relapse during the course of treatment (Carpenter, Schreiber, Church, \& McDowell, 2006; Marissen et al., 2006).

Attention has also been shown to be biased toward stimuli associated with reward outcome in individuals without a reported history of substance abuse (Della Libera \& Chelazzi, 2006, 2009; Hickey, Chelazzi, \& Theeuwes, 2010a, 2010b; Leathers \& Olson, 2012; Peck, Jangraw, Suzuki, Efem, \& Gottlieb, 2009; Raymond \& O’Brien, 2009; Serences, 2008). In particular, we have shown that stimuli become distracting, involuntarily drawing attention (Anderson, Laurent, \& Yantis, 2011a, 2011b, 2012, 2013) and eye movements (Anderson \& Yantis, 2012), when they have been previously paired with reward in an unrelated task (see Anderson, 2013, for a review). This learned attentional bias can persist for extended periods of time without further stimulus-reward pairings (Anderson \& Yantis, 2013), mirroring the persistence of drug-related attentional biases (e.g., Field \& Cox, 2008; Field et al., 2004; Stromark et al., 1997).

Two variables have been shown to be correlated with the magnitude of attentional bias for stimuli associated with non-drug reward: visual working memory (VWM) capacity and impulsiveness (Anderson et al., 2011b; Anderson \& Yantis, 2012). VWM capacity is believed to reflect the efficiency of goal-directed attentional control, with low VWM capacities reflecting greater difficulty resisting distraction by task-irrelevant stimuli (Fukuda \& Vogel, 2009, 2011). Consistent with this notion, individuals with relatively low VWM capacities are particularly susceptible to attentional capture by high-value stimuli (Anderson et al., 2011b; Anderson \& Yantis, 2012). Impulsivity broadly reflects difficulty exercising control over behavior and cognition. Individuals who self-report engaging in more impulsive behavior also show larger attentional biases for reward-associated stimuli (Anderson et al., 2011b).

Addiction is associated with both working memory impairments (e.g., Baldacchino, Balfour, Passetti, Humphris, \& Matthews, 2012; Garavan \& Hester, 2007) and increased impulsiveness (e.g., Hester \& Garavan, 2004; Khurana et al., 2013; Nielsen et al., 2012; Papachristou, Nederkoorn, Havermans, van der Horst, \& Jansen, 2012), and these two characteristics are known risk factors for developing addiction (Dawe \& Loxton, 2004; Khurana et al., 2013). One potential explanation for these risk factors is that they make an individual more susceptible to attentional capture by reward cues, whether these cues are drug-related or not. Individuals whose attention is more powerfully affected by such stimulus-reward associations may be less able to ignore the visual events that signal the potential availability of drug reward, making the addiction more difficult to overcome. In this sense, drug-related attentional biases and their relation to treatment relapse may be reflective of a broader impairment in the ability to ignore any type of reward-associated stimulus.

An important prediction that arises from this account is that individuals who have developed an addiction should show larger attentional biases for stimuli associated with non-drug reward compared to individuals without a history of addiction. In the present study, patients in treatment for addiction and healthy controls first completed a training phase. This consisted of a visual search task in which participants learned over repeated trials that two color-defined targets were associated with monetary reward. Second, participants completed a test phase. This consisted of a different, unrewarded visual search task in which these 
same color stimuli served as irrelevant distractors. We measured the magnitude of response time slowing caused by stimuli associated with non-drug (monetary) reward, an indication of their attentional bias, with the prediction that this bias would be larger in the patient group and related to VWM impairments and greater impulsiveness.

\section{Method}

\section{Participants}

Seventeen patients were recruited from local methadone clinics. Seventeen controls were recruited through newspaper ads and flyer postings in the Baltimore area. Patients and controls did not differ in age, sex, or race ( $p$ 's $>.34)$; they differed in years of education ( $p$ $<.001)$, but not in self-reported monthly household income $(p>.18)$ (see Table 1). All patients had been treated with methadone for at least six months and had been on a stable methadone dose for at least 30 days prior to study enrollment. None of the patients were currently taking any other opioid replacement drug. Patients tested negative for cocaine, amphetamine, methamphetamine, marijuana, opiates, phencyclidine, barbiturates, and benzodiazepines over a minimum of 60 days prior to participation. This was confirmed by urine toxicology reports from the participants' respective clinics, as well as by urine drug testing conducted during the study pre-screening visit and again on the day of testing (Aim Screen MultiDrug 9 by Germaine Laboratories). All controls also tested negative for these same substances during pre-screening and on the day of testing, in addition to testing negative for methadone. Ten patients and five controls were cigarette smokers at the time of study; these participants abstained $0.5-5.5$ hours prior to testing, with the exception of one control who abstained for 7 days prior to testing (abstinence determined from self-report and experimenter observation on the day of testing).

Participants were also screened for the absence of mood, anxiety, and thought disorders using the Structured Clinical Interview for DSM-IV Axis I (SCID) (First, Spitzer, Gibbon, \& Williams, 1996), with consensus ratings obtained by C.L.M with M.L.F. or J.J.R. A detailed history of lifetime drug and alcohol exposure was obtained in both groups using the Lifetime Drug Use Questionnaire (Czermak et al., 2005). Additional exclusionary criteria included a history of neurologic or major medical disorder, alcohol dependence, HIV, and hepatitis $\mathrm{C}$ status that required immediate medication. For patients, testing was conducted 20-24 hours post methadone dose, a time frame well past methadone's peak effects on cognition and physiology, and importantly, a "trough" period when methadone's effects return to baseline (Eissenberg, Stitzer, Bigelow, Buchhalter, \& Walsh, 1999; Walsh, Preston, Stitzer, Cone, \& Bigelow, 1994). The Clinical Opiate Withdrawal Scale (COWS) was conducted immediately after testing to confirm minimal presence of withdrawal symptoms (Tompkins et al., 2009; Wesson \& Ling, 2003); the mean COWS score was 1.6 (SD: 2.5 , range: $0-8$ out of a possible 48 ).

One patient tested positive for cocaine use during the test visit. Use occurred four days before testing. Prior to that, the patient had been drug-negative for 12 months, which is reflected in the reporting of abstinence in Table 1. The difference in value-driven attentional capture and its relation to VWM, along with the difference in impulsiveness, were not affected by the inclusion of this patient.

\section{Apparatus}

Participants completed all components of the experiment in a small, well-lit room. Participants were seated in an office chair behind a large desk, across from which the experimenter was seated. Computer-based tasks were run on a Dell Optiplex 380 and displayed on a Dell E171FP monitor positioned on the desk at a viewing distance of 
approximately $50 \mathrm{~cm}$. All tasks were programmed in Matlab using the Psychophysics Toolbox (Brainard, 1997).

\section{Impulsiveness Questionnaire}

Prior to completing the computer-based tasks, participants completed the Barratt Impulsiveness Scale (BIS-11) (Patton, Stanford, \& Barratt, 1995). The BIS-11 contains 30 statements about behavior that participants report how often they engage in, using a fouralternative forced-choice response. To ensure that all participants understood each statement regardless of reading ability, the BIS-11 was administered orally by the experimenter.

\section{Visual Working Memory Task}

Prior to completing the attentional capture task, all participants completed a color changedetection task (Luck \& Vogel, 1997). On each of 120 trials, a memory array was presented following a $500 \mathrm{~ms}$ fixation period and consisted of the presentation of 2, 4, or 6 colored squares $\left(1.38^{\circ} \times 1.38^{\circ}\right)$ on a grey background. The color of the squares were selected from red, green, yellow, blue, cyan, orange, black, purple, and brown without replacement; each square was separated by at least $2.06^{\circ}$ center-to-center. The memory array was presented for $100 \mathrm{~ms}$ and was followed by a blank $900 \mathrm{~ms}$ retention interval. After the retention interval, a single colored square was presented in a position previously occupied by a square in the memory array. This probe square was either the same or different in color as the square that had been previously presented in its location (equally-often). Participants indicated whether they thought the color of the probed square had changed via an unspeeded, two alternative forced-choice key press, pressing standard keyboard letter " $\mathrm{m}$ " for reporting a change and "z" for reporting no change (these keys were labeled "s" for same and "diff" for different to indicate the corresponding response). No feedback was provided during the task.

Immediately preceding the task, all participants completed 24 practice trials, randomly generated using the aforementioned parameters, during which feedback concerning accuracy was provided to ensure understanding of task instruction.

\section{Attentional Capture Task}

Training phase-The training phase consisted of visual search for a target circle among five non-target circles (see Figure 1A). The target circle was unpredictably red or green. Participants reported the orientation of a bar contained within the target as either vertical or horizontal via a key press, with the " $\mathrm{z}$ " key for vertical and the " $\mathrm{m}$ " key for horizontal (the key labels also contained the corresponding oriented bar). Bars contained within the nontarget circles were randomly oriented at $45^{\circ}$ to the left or to the right.

Each visual search array was followed by feedback indicating monetary reward. If participants responded correctly on the trial, a small amount of money was added to a running total that participants were informed they would be paid at the completion of the experiment. Importantly, one of the two target colors was followed by a comparatively high reward of $10 \notin$ on $80 \%$ of the trials it was correctly reported, and by a comparatively low reward of $2 \phi$ on the remaining $20 \%$ (high-reward target); for the other color target, these contingencies were reversed (low-reward target). If participants did not respond correctly, the feedback indicated that $0 \notin$ had been earned. Red served as the high-reward color for half of the participants (eight patients and nine controls).

Each trial in the training phase consisted of a fixation display for 400, 500, or $600 \mathrm{~ms}$ (randomly determined), which was followed by the search array for either $1200 \mathrm{~ms}$ or until a response was made. A $250 \mathrm{~ms} 1000 \mathrm{~Hz}$ beep informed participants if they failed to execute a response during the $1200 \mathrm{~ms}$ deadline, and such trials were scored as errors. The search array was followed by a blank screen for $1000 \mathrm{~ms}$, the reward feedback display for $1500 \mathrm{~ms}$, 
and then by a blank $1000 \mathrm{~ms}$ inter-trial-interval (ITI). The background of the screen was black, the fixation cross and oriented bars were white, and the non-target circles were drawn from the colors orange, blue, cyan, white, pink, and yellow without replacement. The fixation cross was $1.09^{\circ} \times 1.09^{\circ}$ and each circle was $3.95^{\circ} \times 3.95^{\circ}$, positioned in one of six locations along an imaginary circle with a radius of $8.12^{\circ}$.

The training phase consisted of 240 trials, half of which contained a red target and half of which contained a green target. These trials were broken into four blocks of 60 trials, with a mandatory $30 \mathrm{sec}$ break between blocks. Each color target appeared in each location equally-often, and each color target contained a vertical and horizontal bar equally often. The order of trials was randomized for each participant. The training phase began with 40 practice trials, randomly generated using the aforementioned parameters.

Test phase-The test phase consisted of visual search for a unique shape, either a circle among diamonds or a diamond among circles (see Figure 1B). The color of the shapes was now irrelevant to the task, and participants were informed of this. Importantly, one of the non-target shapes was occasionally rendered in the color of a formerly reward-predictive target. On $25 \%$ of the trials, one of the non-targets was green and on a different $25 \%$ of the trials, one of the non-targets was red; these red and green shapes constituted the valuable distractors. On the remaining $50 \%$ of the trials, none of the shapes were red or green (distractor-absent trials). The targets were never red or green.

Participants made the same judgment concerning the orientation of the bar contained within the target, indicating whether it was vertical or horizontal. Importantly, they were no longer provided a monetary reward for doing so. Feedback following the search array only informed participants if their prior response was correct or not, with a check mark for correct responses and an "X" for incorrect responses.

Each trial consisted of a fixation display for 400, 500, or $600 \mathrm{~ms}$ (randomly determined), a search array for $1500 \mathrm{~ms}$ or until a response was made, a feedback display for $1000 \mathrm{~ms}$, and a $500 \mathrm{~ms}$ blank ITI. The positions and size of the stimuli and the colors used were identical to the training phase, and a beep informed participants if a response was not made within $1500 \mathrm{~ms}$. The target was equally-often a diamond among circles and a circle among diamonds, and the target shape and its location were unrelated to the presence, color, and position of the distractors. The test phase also consisted of 240 trials, randomly ordered and separated into four blocks, and it began with 28 practice trials randomly generated using the same parameters.

Assessment of awareness-Following the test phase, awareness of the reward value of targets during the training phase was assessed. Participants were asked to indicate whether they thought (a) "the red circle was usually worth more than the green" (b) "the green circle was usually worth more than the red" or (c) "the two circles were usually worth about the same". Participants who answered (c) were subsequently informed that one color circle was in fact usually worth more than the other, and were asked to guess which color it was.

\section{Data Analysis}

Only response time (RT) on correct trials was considered in the analyses of RT. RTs exceeding 3 standard deviations of the mean of a given condition for a given participant were trimmed. RTs faster than $200 \mathrm{~ms}$ ( $<1 \%$ of trials) were considered anticipations and were not analyzed. Two participants ( 1 patient and 1 control) had difficulty meeting the response deadline and could not initially perform at or above chance level in the visual search task for the first two blocks; for these participants, these blocks were considered additional practice and were not analyzed. 
For the VWM task, accuracy was recorded and VWM capacity was calculated as the number of items remembered using a standard formula that accounts for the probability of guessing correctly (Cowen, 2001). Two patients performed at or below chance in the VWM task for set sizes 4 and 6, but performed accurately at set size 2; only data from set size 2 were considered for these participants (based on feedback from these patients, we reason that they found the larger set sizes too difficult and felt forced to guess at these set sizes). All of the correlations with VWM remain significant when all three set sizes are considered for these patients.

Impulsiveness was measured as the total score obtained on the BIS-11 (possible range: 30 120). We also broke impulsiveness down into the three sub-scales of the BIS-11 (Attentional Impulsiveness, Motor Impulsiveness, and Non-planning Impulsiveness).

In assessing awareness of the stimulus-reward contingencies, participants who correctly answered (a) or (b) to the awareness question were scored as being aware of the relationship, and participants who answered (c) as unaware. For participants who answered (c), the guessing rate was obtained from their response to the follow-up forced-choice question.

We compared RT and accuracy between high- and low-reward targets during the training phase and across the three distractor conditions (absent, low-value, and high-value) during the test phase separately for patients and controls. Sex was included as an additional between-subjects factor to assess whether any of the reported effects were sex-specific. Value-driven attentional capture (slowing in RT caused by the high-value distractor in the test phase) was compared to VWM capacity, impulsiveness, and several demographic measures (including education, household income, and measures of drug use history) using Pearson correlations.

\section{Results}

\section{Training Phase}

Mean RT in the training phase was subjected to a $2 \times 2 \times 2$ mixed design analysis of variance (ANOVA), with target value (high vs low) as a within-subjects factor and patient status and sex as between-subjects factors. Participants were faster to report the high-reward target $($ mean $=706 \mathrm{~ms})$ compared to the low-reward target $($ mean $=721 \mathrm{~ms})$, though not significantly so $[F(1,30)=2.69, p=.112]$. Target value did not interact with patient status $[F(1,30)=1.98, p=.170]$, suggesting that both patients and controls were similarly motivated by the monetary rewards. Nor did target value interact with sex $(F<1)$, and the three-way interaction was not significant $[F(1,30)=1.45, p=.238]$. There was a main effect of patient status in which patients (mean $=747 \mathrm{~ms}$ ) were overall slower than the controls $($ mean $=680 \mathrm{~ms})[F(1,30)=7.42, p=.011]$, and a main effect of sex in which males were overall faster than females (mean difference $=63 \mathrm{~ms}$ ) $[F(1,30)=6.31, p=.018]$, but no interaction between sex and patient status $[F(1,30)=1.13, p=.295]$. Similar results were obtained using accuracy as the dependent measure [target value: $F(1,30)=3.46, p=.073$; patient status: $F(1,30)=15.42, p<.001$; other $p$ 's $>.15]$. Mean accuracy was $83.4 \%$ for the patients and $93.7 \%$ for the controls. Reflecting accuracy, mean earnings in the task were $\$ 12.10$ for patients and $\$ 13.51$ for controls $[t(32)=3.69, p=.001]$.

\section{Test Phase}

Mean RT in the test phase was subjected to a $3 \times 2 \times 2$ mixed design ANOVA, with distractor condition (absent, low-value, high-value) as a within-subjects factor and patient status and sex as between-subjects factors. There was a main effect of distractor condition [Figure $2, F(2,60)=9.92, p<.001]$, reflecting attentional capture by the previously reward- 
associated distractors; this effect did not differ based on which color served as the highreward color during training or current smoking status $(F$ 's $<1)$, so we collapsed across these factors. There was also a main effect of patient status in which the patients performed significantly slower than the controls overall $[F(1,30)=10.03, p=.004]$; the main effect of $\operatorname{sex}[F(1,30)=3.06, p=.091]$ and the interaction between sex and patient status $(F<1)$ were not significant.

Importantly, there was a significant interaction between patient status and distractor condition $[F(2,60)=4.37, p=.017]$. We defined value-driven attentional capture as the slowing in RT caused by the high-value distractor compared to distractor-absent trials, as we have done in prior studies (Anderson et al., 2011b; Anderson and Yantis, 2012). A planned comparison revealed that the magnitude of value-driven attentional capture was significantly greater in the patients than in the controls $[t(32)=2.75, p=.010]$; the magnitude of slowing caused by the low-value distractor was also greater in the patients, but this difference did not reach significance $[t(32)=1.63, p=.113]$. Distractor condition also interacted significantly with sex $[F(2,60)=6.59, p=.003]$, such that value-driven attentional capture was greater in males than in females (mean difference $=23 \mathrm{~ms}$ ). The three-way interaction was not significant $(F<1)$, however, indicating that the observed sex difference was present in both the patients and the controls.

The same $3 \times 2 \times 2$ mixed design ANOVA was also run on accuracy, which revealed an interaction between sex and patient status $[F(1,30)=4.49, p=.043]$ in which male patients were more accurate than female patients while the reverse was true of controls; otherwise, there were no main effects or interactions (all $p$ 's $>.09$ ). Mean accuracy for the patients was $84.2 \%, 85.5 \%$, and $82.7 \%$ across the absent, low-value, and high-value distractor conditions, respectively, and $89.1 \%, 89.9 \%$, and $88.4 \%$ for the controls.

\section{Predicting Attentional Bias from Individual Difference Measures}

VWM capacity was first subjected to a $2 \times 2$ ANOVA, with patient status and sex as between-subjects factors. A main effect of patient status revealed that the VWM capacity of the controls (mean $=2.01$ ) was larger than that of the patients (mean $=1.54)[F(1,30)=6.01$, $p=.020]$. There was no main effect of $\operatorname{sex}(F<1)$, nor did sex interact with patient status $[F(1,30)=2.06, p=.162]$.

The magnitude of value-driven attentional capture (slowing in RT caused by the high-value distractor) was negatively correlated with VWM capacity across all participants $(r=-.518$, $p=.002$ ), replicating previous findings (Anderson et al., 2011b; Anderson and Yantis, 2012). Thus, individuals who exhibited the least effective attentional control, as evidenced by performance in the VWM task, were also the most affected by the distractors. This correlation was present specifically within the patient group $(r=-.697, p=.002)$, with VWM capacity predicting which patients experienced the greatest difficulty ignoring reward-related stimuli, but was not evident within the control group $(r=-.046, p=.860)$ (see Figure 3).

The attentional bias for the previously reward-associated distractors was not correlated with impulsiveness as measured using the BIS-11 $(p>.89)$, nor did it correlate with any of the BIS-11 subscales ( $p$ 's $>.39$ ). However, a $2 \times 2$ ANOVA with patient status and sex as between-subjects factors revealed a main effect of patient status in which the patients (mean $=62.9)$ were significantly more impulsive than the controls $($ mean $=54.1)[F(1,30)=8.52, p$ $=.007]$; the main effect of sex and the interaction between sex and patient status were not significant (both $F$ 's $<1$ ). 
Value-driven attentional capture was not significantly correlated with years of education or household income in either the patients or controls ( $p$ 's > .24), nor did it correlate with RT on distractor-absent trials $(p$ 's $>.17)$. The latter indicates that value-driven attentional capture was not simply greater for individuals who were overall slower at performing the task, and this is consistent with prior reports (Anderson et al., 2011b). Within the patients, value-driven attentional capture was not significantly correlated with duration of abstinence from illicit drugs $(p>.33)$, age of first illicit drug use $(p>.58)$, lifetime opioid exposure ( $p$ $>.62)$, or current methadone dose $(p>.70)$.

\section{Awareness}

During the training phase, the relationship between target color and reward magnitude was probabilistic (80/20). This rendered the relationship between the two as implicit for most of our participants. During the post-experimental assessment of awareness, four patients and three controls correctly indicated the color that was associated with higher overall reward in the training phase, demonstrating explicit awareness of the relationship. The remaining participants indicated that both colors were worth about the same (i.e., option C) and were scored as unaware. Of the participants who were unaware of the relationship between color and reward, neither patients nor controls guessed the correct color above chance ( $p$ 's $>.13$ ), and guessing rates did not differ between groups $\left(\chi^{2}<1\right)$. The effect of the previously reward-associated distractors on performance in the test phase did not differ based on whether participants were aware of the relationship between color and reward during training or whether unaware participants guessed correctly $(p$ 's $>.19)$.

\section{Discussion}

Our results show that individuals in treatment for drug addiction, in this case opioid dependence, were more distracted by stimuli previously associated with non-drug reward compared to individuals without a history of addiction. Thus, we demonstrate that rewardrelated attentional biases in addiction are not limited to the influence of drug reward and extend to stimuli associated with other forms of reward. This finding suggests that attentional biases for drug-related stimuli (Carpenter et al., 2006; Field \& Cox, 2008; Field et al., 2004; Lubman et al., 2000; Marissen et al., 2006; Mogg et al., 2003; Stromark et al., 1997) may be reflective of a broader impairment in reward-driven attentional control; addiction is associated with an impaired ability to ignore reward-related stimuli more generally, which may contribute to the attention-capturing quality of stimuli associated with drug reward.

It is unclear whether this increased bias to attend to reward-associated stimuli precedes the onset of addiction or rather is the result of cognitive changes brought on by substance use. One possibility is that individuals who are particularly prone to attentional capture by reward-associated stimuli are more likely to develop drug-related attentional biases, being less capable of ignoring drug-related stimuli during the early stages of drug usage. Another possibility is that the attentional system becomes hypersensitive to reward-related influences as a consequence of either chronic drug usage or acute effects of a drug (in this case, methadone), leading to heightened attentional capture by stimuli associated with drug and non-drug reward. Of course, both factors could be contributing to the present findings. All patients had abstained from methadone for 20-24 hours prior to participation and their current methadone dose did not correlate with value-driven attentional capture, making it unlikely that the increased attentional bias in the patients was entirely a function of methadone's acute pharmacological effects. In fact, there is evidence that methadone maintenance treatment can actually improve goal-directed attention allocation and reduce drug-related attentional bias in patients with opioid dependence (Nejati, Nejati, \& 
Mohammadi, 2011). Importantly, the relationship between drug-related attentional biases in addiction and treatment outcome (Carpenter et al., 2006; Marissen et al., 2006) suggests that changes in reward-related attentional biases would be clinically relevant at either stage of onset.

Our findings also suggest a potential mechanism by which working memory impairment and greater impulsiveness could contribute to the development and maintenance of addiction. Prior research has shown that individuals with lower VWM capacity and higher impulsiveness are particularly susceptible to attentional capture by reward-associated stimuli (Anderson et al., 2011b; Anderson \& Yantis, 2012). Consistent with these prior findings, VWM capacity predicted the magnitude of attentional capture across all participants and specifically within the patient group, and patients were significantly more impulsive than controls. The present results support the notion that working memory impairments and greater impulsiveness are associated with greater difficulty ignoring stimuli associated with both drug and non-drug reward.

In our sample, the patients had significantly fewer years of education than the controls. This is unsurprising, given the destructive consequences of substance abuse. It is welldocumented that the use of illicit substances is associated with reduced educational completion (e.g., Townsend, Flisher, \& King, 2007). All but one of the patients in the present study had begun using illicit substances before the age of twenty, most before the age of sixteen. It is unclear how reduced education would directly impact an individual's sensitivity to the effects of reward on attention, and years of education did not correlate with the magnitude of value-driven attentional capture in either the patients or the controls. The latter suggests that attentional bias for reward-related stimuli is not a direct function of years of education. Never-the-less, we cannot completely rule out education as playing a role in biasing attention in favor of reward-predictive stimuli. Given the clinical relevance of attentional biases in addiction (Carpenter et al., 2006; Marissen et al., 2006), however, characterizing the nature of this bias is important regardless of its relation to education.

It is also worth noting that the observed attentional biases for previously reward-predicting stimuli were evident in the absence of currently available rewards. No monetary rewards were delivered during the test phase, when value-driven attentional capture was assessed. Therefore, the present findings cannot be explained by expectations concerning the current value of stimuli; rather, these findings reflect the attentional consequences of prior reward learning, which are more pronounced in patients in treatment for addiction than in individuals without a history of addiction.

A potential concern is that the patients may have placed greater value on money than the controls did due to a more impoverished lifestyle. However, patients and controls did not significantly differ in monthly household income, nor did income correlate with valuedriven attentional capture in either the patients or the controls. Future research will be needed to extend our findings beyond monetary reward to other forms of non-drug reward.

Future research should also be aimed at investigating whether non-drug reward-related attentional biases predict future substance abuse and subsequent treatment outcome, as has been found with drug-related attentional biases (Carpenter et al., 2006; Marissen et al., 2006). A predictive relationship would suggest that general reward-related attentional biases play a causal role in establishing and maintaining addictive behaviors. Many drug-addicted individuals must overcome risks in acquiring a substance of abuse. Future research could investigate the extent to which addiction is associated with reduced attention to punishmentrelated stimuli. 
The findings of the present study contribute to the understanding of attentional biases observed in addiction. We show that addiction is associated with a broad impairment in the ability to ignore reward-associated stimuli that extends beyond drug reward. A better understanding of how reward and attention interact in addiction may lead to insights into how to improve treatment and prevention.

\section{Acknowledgments}

This research was supported by U.S. National Institutes of Health grant R01-DA013165 to S.Y., career development award K01-DA030442 to C.L.M., and fellowship F31-DA033754 to B.A.A. The funding sources played no role in the study beyond financial support.

\section{References}

Anderson BA. A value-driven mechanism of attentional selection. Journal of Vision. 2013; 13(3):7, 1 16.

Anderson BA, Laurent PA, Yantis S. Reward predictions bias attentional selection. Frontiers in Human Neuroscience. 2013; 7:262.

Anderson BA, Laurent PA, Yantis S. Generalization of value-based attentional priority. Visual Cognition. 2012; 20:647-658.

Anderson BA, Laurent PA, Yantis S. Learned value magnifies salience-based attentional capture. PLoS ONE. 2011a; 6:e27926.

Anderson BA, Laurent PA, Yantis S. Value-driven attentional capture. Proceedings of the National Academy of Science of the United States of America. 2011b; 108:10367-10371.

Anderson BA, Yantis S. Persistence of value-driven attentional capture. Journal of Experimental Psychology: Human Perception and Performance. 2013; 39:6-9.

Anderson BA, Yantis S. Value-driven attentional and oculomotor capture during goal-directed, unconstrained viewing. Attention, Perception, and Psychophysics. 2012; 74:1644-1653.

Baldacchino A, Balfour DJK, Passetti F, Humphris G, Matthews K. Neuropsychological consequences of chronic opioid abuse: a quantitative review and meta-analysis. Neuroscience and Biobehavioral Reviews. 2012; 36:2056-2068. [PubMed: 22771335]

Berridge KC. From prediction error to incentive salience: mesolimbic computation of reward motivation. European Journal of Neuroscience. 2012; 35:1124-1143.

Berridge KC, Robbinson TE. What is the role of dopamine in reward: hedonics, learning, or incentive salience? Brain Research Reviews. 1998; 28:309-369.

Brainard DH. The psychophysics toolbox. Spatial Vision. 1997; 10:433-436. [PubMed: 9176952]

Carpenter KM, Schreiber E, Church S, McDowell D. Drug Stroop performance: relationships with primary substance of use and treatment outcome in a drug-dependent outpatient sample. Addictive Behaviors. 2006; 31:174-181. [PubMed: 15913898]

Cowen N. The magical number 4 in short-term memory: a reconsideration of mental storage capacity. Behavioral and Brain Sciences. 2001; 24:87-114. [PubMed: 11515286]

Czermak C, Lehofer M, Gasser-Steiner P, Ettinger S, Lemonis L, Rohrhofer A, Legl T, Leibmann PM. Test-retest reliability of a lifetime drug use questionnaire. Addictive Behaviors. 2005; 30:361-368. [PubMed: 15621408]

Dawe S, Loxton NJ. The role of impulsivity in the development of substance use and eating disorders. Neuroscience and Biobehavioral Reviews. 2004; 28:343-351.

Della Libera C, Chelazzi L. Learning to attend and to ignore is a matter of gains and losses. Psychological Science. 2009; 20:778-784. [PubMed: 19422618]

Della Libera C, Chelazzi L. Visual selective attention and the effects of monetary reward. Psychological Science. 2006; 17:222-227.

Eissenberg T, Stitzer ML, Bigelow GE, Buchhalter AR, Walsh SL. Relative potency of levo-alphaacetylmethadol and methadone in humans under acute dosing conditions. Journal of Pharmacology and Experimental Therapeutics. 1999; 289:936-945.

Exp Clin Psychopharmacol. Author manuscript; available in PMC 2014 December 01. 
Fukuda K, Vogel EK. Individual differences in recovery time from attentional capture. Psychological Science. 2011; 22:361-368.

Fukuda K, Vogel EK. Human variation in overriding attentional capture. Journal of Neuroscience. 2009; 29:8726-8733.

Field M, Cox WM. Attentional bias in addictive behaviors: a review of its development, causes, and consequences. Drug and Alcohol Dependence. 2008; 97:1-20. [PubMed: 18479844]

Field M, Mogg K, Zetteler J, Bradley BP. Attentional biases for alcohol cues in heavy and light social drinkers: the roles of initial orienting and maintained attention. Psychopharmacology (Berlin). 2004; 173:116-123. [PubMed: 14663552]

First, MB.; Spitzer, RL.; Gibbon, M.; Williams, JBW. Structured Clinical Interview for DSM-IV Axis I Disorders, Clinical Version (SCID-CV). Washington: American Psychiatric Press; 1996.

Garavan H, Hester R. The role of cognitive control in cocaine dependence. Neuropsychology Review. 2007; 17:337-345.

Hester R, Garavan H. Executive dysfunction in cocaine addiction: evidence for discordant frontal, cingulate, and cerebellar activity. Journal of Neuroscience. 2004; 24:11017-11022.

Hickey C, Chelazzi L, Theeuwes J. Reward changes salience in human vision via the anterior cingulate. Journal of Neuroscience. 2010a; 30:11096-11103.

Hickey C, Chelazzi L, Theeuwes J. Reward guides vision when it's your thing: trait reward-seeking in reward-mediated visual priming. PLoS ONE. 2010b; 5:e14087. [PubMed: 21124893]

Khurana A, Romer D, Betancourt LM, Brodsky NL, Giannetta JM, Hurt H. Working memory ability predicts trajectories of early alcohol use in adolescents: the mediational role of impulsivity. Addiction. 2013; 108:506-515.

Leathers ML, Olson CR. In monkeys making value-based decisions, lip neurons encode cue salience and not action value. Science. 2012; 338:132-135. [PubMed: 23042897]

Luck SJ, Vogel EK. The capacity of visual working memory for features and conjunctions. Nature. 1997; 390:279-281. [PubMed: 9384378]

Lubman DI, Peters LA, Mogg K, Bradley BP, Deakin JFW. Attentional bias for drug cues in opiate dependence. Psychological Medicine. 2000; 30:169-175.

Marissen MAE, Franken IHA, Waters AJ, Blanken P, van den Brink W, Hendriks VM. Attentional bias predicts heroin relapse following treatment. Addiction. 2006; 101:1306-1312. [PubMed: 16911730]

Mogg K, Bradley BP, Field M, De Houwer J. Eye movements to smoking-related pictures in smokers: relationship between attentional biases and implicit and explicit measures of stimulus valence. Addiction. 2003; 98:825-836. [PubMed: 12780371]

Nejati M, Nejati V, Mohammadi MR. Selective attention and drug-related attention bias in methadone maintenance patients. Acta Medica Iranica. 2011; 49:814-817. [PubMed: 22174171]

Nielsen DA, Ho A, Bahl A, Varma P, Kellogg S, Borg L, et al. Former heroin addicts with and without a history of cocaine dependence are more impulsive than controls. Drug and Alcohol Dependence. 2012; 124:113-120.

Nickolaou K, Field M, Critchley H, Duka T. Acute alcohol effects on attentional bias are mediated by subcortical areas associated with arousal and salience attribution. Neuropsychopharmacology. 2013; 38:1365-1373.

Nickolaou K, Field M, Duka T. Alcohol-related cues reduce cognitive control in social drinkers. Behavioral Pharmacology. 2013; 24:29-36.

Papachristou H, Nederkoorn C, Havermans R, van der Horst M, Jansen A. Can't stop the craving: the effect of impulsivity on cue-elicited craving for alcohol in heavy and light social drinkers. Psychopharmacology (Berlin). 2012; 219:511-518.

Patton JH, Stanford MS, Barratt ES. Factor structure of the Barratt Impulsiveness Scale. Journal of Clinical Psychology. 1995; 51:768-774.

Peck CJ, Jangraw DC, Suzuki M, Efem R, Gottlieb J. Reward modulates attention independently of action value in posterior parietal cortex. Journal of Neuroscience. 2009; 29:11182-11191.

[PubMed: 19741125] 
Raymond JE, O'Brien JL. Selective visual attention and motivation: the consequences of value learning in an attentional blink task. Psychological Science. 2009; 20:981-988.

Serences JT. Value-based modulations in human visual cortex. Neuron. 2008; 60:1169-1181.

Stromark KM, Field NP, Hugdahl K, Horowitz M. Selective processing of visual alcohol cues in abstinent alcoholics: an approach-avoidance conflict? Addictive Behaviors. 1997; 22:509-519. [PubMed: 9290860]

Tompkins DA, Bigelow GE, Harrison JA, Johnson RE, Fudala PJ, Strain EC. Concurrent validation of the Clinical Opiate Withdrawal Scale (COWS) and single-item indices against the Clinical Institute Narcotic Assessment (CINA) opioid withdrawal instrument. Drug and Alcohol Dependence. 2009; 105:154-159.

Townshend JM, Duka T. Attentional bias associated with alcohol cues: Differences between heavy and occasional social drinkers. Psychopharmacology (Berlin). 2001; 157:67-74.

Townsend L, Flisher AJ, King G. A systematic review of the relationship between high school dropout and substance use. Clinical Child and Family Psychology Review. 2007; 10:295-317.

Walsh SL, Preston KL, Stitzer ML, Cone EJ, Bigelow GE. Clinical pharmacology of buprenorphine: ceiling effects at high doses. Clinical Pharmacology and Therapeutics. 1994; 55:569-580. [PubMed: 8181201]

Wesson DR, Ling W. The Clinical Opiate Withdrawal Scale (COWS). Journal of Psychoactive Drugs. $2003 ; 35: 253-259$.

Exp Clin Psychopharmacol. Author manuscript; available in PMC 2014 December 01. 
A

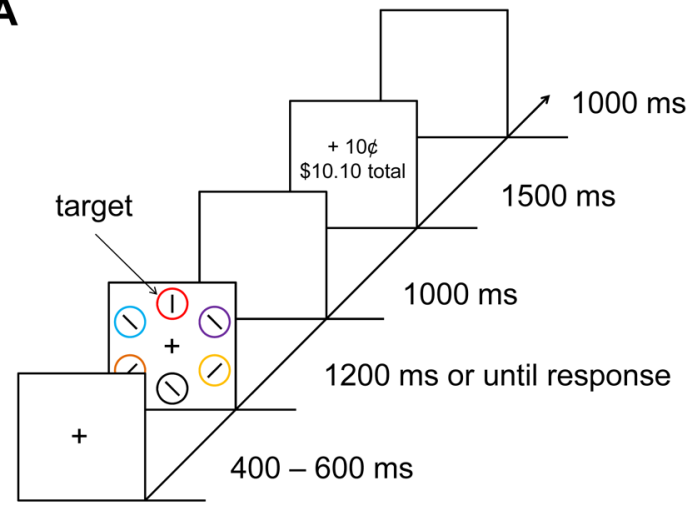

B

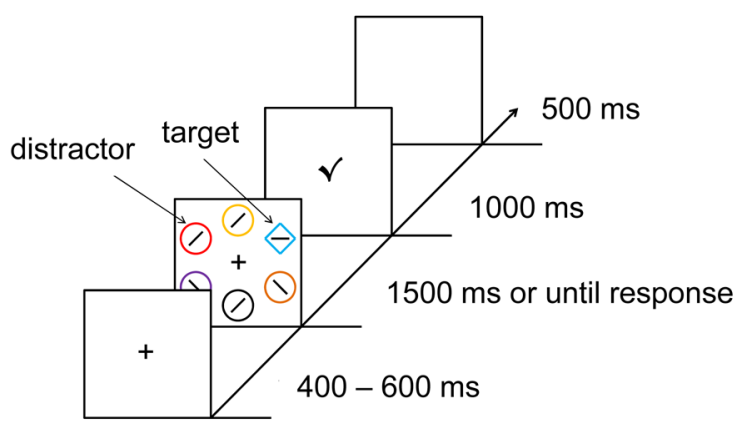

Figure 1.

Sequence of events and time course for a trial during the training phase (A) and test phase (B) of the visual search task. During the training phase, patients and controls searched for a target circle that was unpredictably red or green, and received a monetary reward for correctly reporting the orientation of a bar contained within the target. During the test phase, participants searched for a target defined as the unique shape (e.g., diamond among circles) and no monetary rewards were provided. On a subset of the trials, one of the non-target shapes was rendered in the color of a formerly reward-predictive target (i.e., red or green), which served as the reward-associated distractor. 


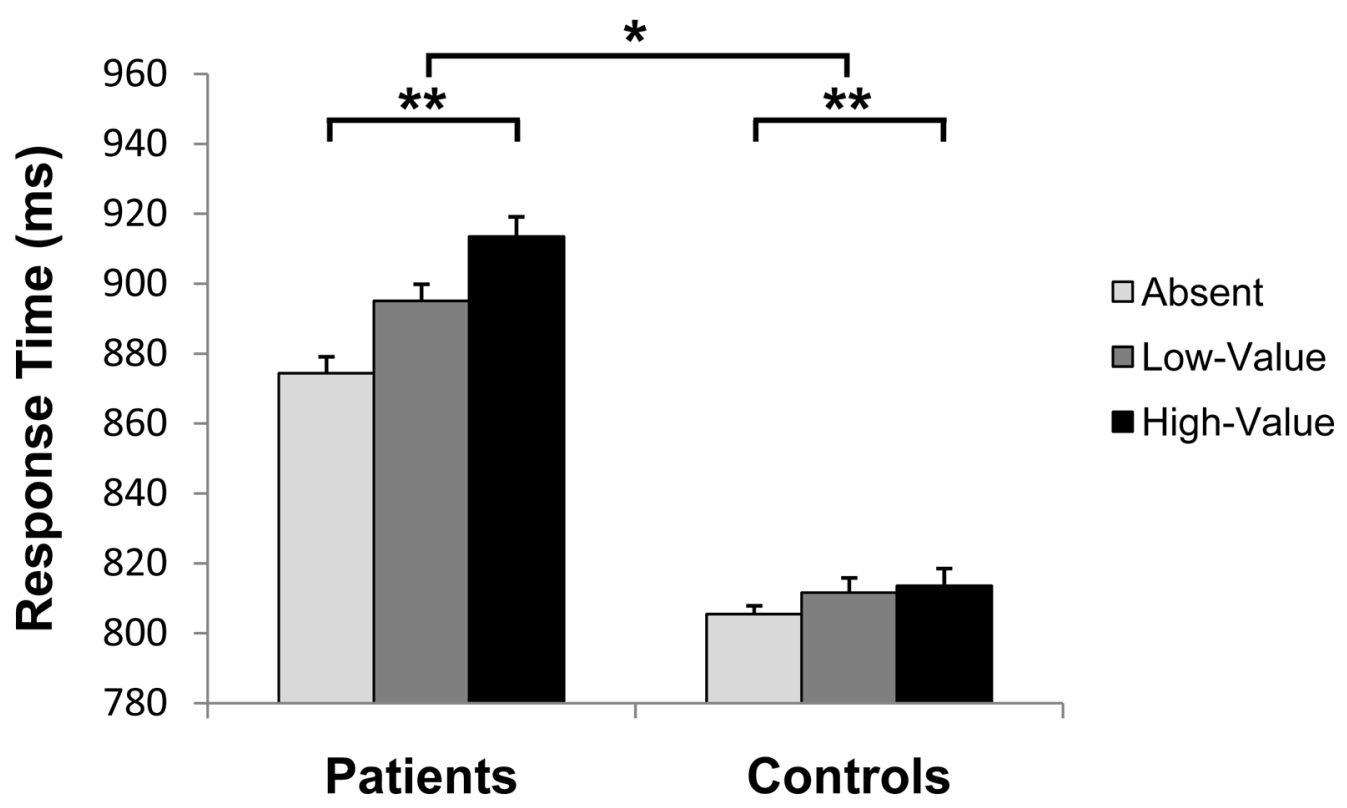

Figure 2.

Response time across the three distractor conditions in the test phase for patients and controls. There was a main effect of distractor condition in which participants were significantly slower to report the target when a high-value distractor was present compared to when no distractor was present ("absent"), indicating value-driven attentional capture $(* * \mathrm{p}<.001)$. The magnitude of this slowing was significantly greater in the patients than in the controls $(* \mathrm{p}<.01)$, indicating greater susceptibility to attentional capture by previously reward-associated stimuli. Error bars reflect the within-subjects standard error of the mean. 

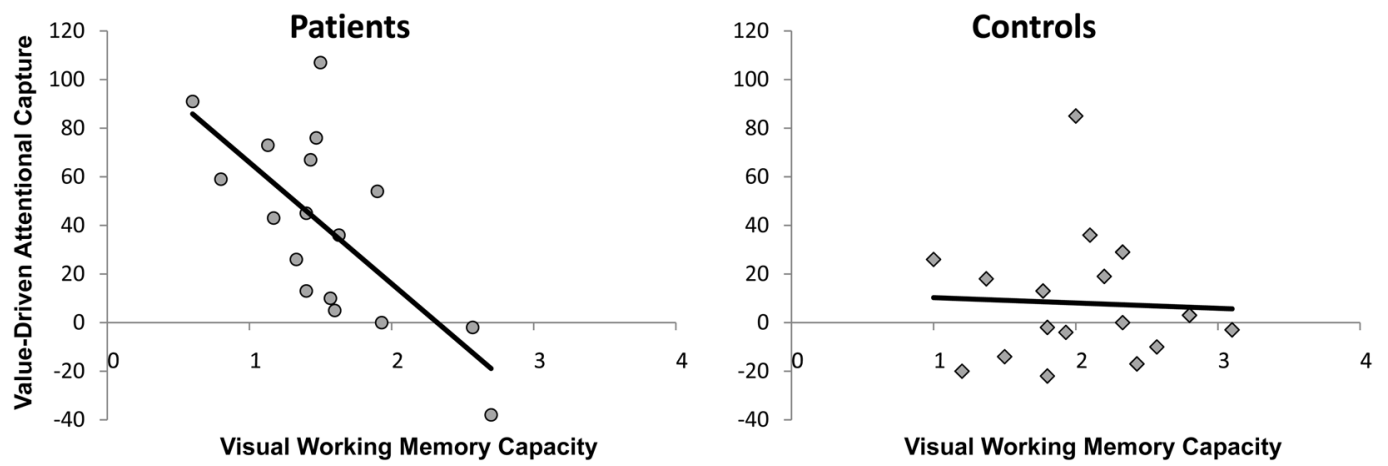

Figure 3.

Correlation between value-driven attentional capture (slowing in RT caused by the highvalue distractor) and visual working memory capacity separately for patients $(r=-.697, p$ $=.002)$ and controls $(r=-.046, p=.860)$. 


\section{Table 1}

Demographics and lifetime exposure to substances $^{1}$ (exposure calculated from data provided in a structured interview) for patients and controls. Standard deviations are in parentheses.

\begin{tabular}{|c|c|c|}
\hline & Patients & Controls \\
\hline Male:Female & $9: 8$ & $9: 8$ \\
\hline Age $(y)$ & $44.4(10.0)$ & $42.9(11.0)$ \\
\hline Education $(y)^{*}$ & $11.5(1.3)$ & $14.7(1.8)$ \\
\hline Monthly Household Income $(\text { US } \$)^{2}$ & $1431(1311)$ & 2328 (2344) \\
\hline Methadone Dose (mg) & $77.9(33.4)$ & N/A \\
\hline Lifetime Alcohol Exposure (l) & $5117.2(8450.2)$ & $1412.8(2850.5)$ \\
\hline Lifetime Nicotine Exposure (packs of cigarettes) & $5058.7(6959.8)$ & $1810.0(4859.1)$ \\
\hline Lifetime Marijuana Exposure $(g)$ & $3040.4(7905.8)$ & $1908.0(5246.8)$ \\
\hline Lifetime Opioid Exposure $(g)^{*}$ & $3864.2(4057.1)$ & $0(0)$ \\
\hline Lifetime Cocaine Exposure $(g)^{*}$ & $1552.1(1343.7)$ & $32.0(131.5)$ \\
\hline Abstinence from Illicit Drug Use (mos) $)^{3}$ & $20.1(21.0)$ & $178.0(179.6)$ \\
\hline
\end{tabular}

${ }^{1}$ Substances listed include those endorsed by at least five participants. Additional substances were reported, but amounts were considered to be negligible for overall group comparisons. These included hashish, amphetamines, LSD, Ecstasy, benzodiazepines, barbiturates, and inhalants.

2

Excludes one control, whose monthly household income exceeded the mean of all other participants by over 52 standard deviations.

${ }^{3}$ Calculated only for those who reported prior use of any illicit substance ( 8 of 17 controls). Range: $3-72$ mos for patients, $2-420$ mos for controls.

Denotes significant difference between groups $(\mathrm{p}<.05)$. 